\author{
Proceedings of the ASME 2020 \\ Dynamic Systems and Control Conference \\ DSCC2020 \\ October 4-7, 2020, Pittsburgh, PA, USA
}

DSCC2020-3107

\title{
CONTROL DESIGN FOR AN EMULATOR OF MECHATRONIC POWERTRAIN DYNAMICS: A CASE STUDY
}

\author{
Laurens Jacobs $^{1,3}$, Dennis Janssens ${ }^{2,3}$, Jan Croes $^{2,3}$, Wim Desmet ${ }^{2,3}$, Jan Swevers ${ }^{1,3}$ \\ ${ }^{1}$ MECO Research Team \\ ${ }^{2}$ LMSD Division \\ ${ }^{3}$ DMMS Lab \\ Dept. of Mechanical Engineering \\ KU Leuven \\ 3001 Leuven, Belgium \\ $\langle$ firstname〉.〈lastname〉@kuleuven.be \\ Dept. of Mechanical Engineering \\ KU Leuven \\ 3001 Leuven, Belgium \\ $\langle$ firstname〉.〈lastname〉@kuleuven.be \\ Flanders Make \\ 3001 Leuven, Belgium
}

\begin{abstract}
This paper discusses a detailed design procedure of a linear torque controller for an in-house developed lab-scale powertrain emulator. This set-up was developed for the validation of new developments in virtual torque sensing, and thus requires decent torque tracking performance in order to simulate real drivetrain dynamics sufficiently accurate. Standard proportional-integral (PI) controllers are not able to effectively compensate for potential flexible modes of the set-up, and an $\mathscr{H}_{\infty}$ control approach is proposed. The different design steps then comprise experiment design, identification, feedback controller design using $\mathscr{H}_{\infty}$ loop shaping, and inverse-based feedforward controller design. Experimental results demonstrate the obtained torque tracking performance.
\end{abstract}

\section{INTRODUCTION}

Mechatronic powertrains have become omnipresent in a wide variety of technological fields. Well-known examples are found in vehicle technology (e.g. hybrid cars), manufacturing (e.g. weaving looms) and energy conversion (e.g. wind turbines). Apart from being compact and light-weight, one of their main advantages is the possibility of active and highly dynamic control through high-end control of one (or multiple) electric drive(s). These drives can hence directly adapt their delivered power to changing load dynamics without requiring mechanical transmissions or inertias in the drivetrain. An inherent challenge related to the use of such direct drive systems, however, is the strong interaction between the load dynamics and the drive dynamics. Therefore, it is often useful to have torque measurements at one's disposal while designing, prototyping or analyzing powertrains, in order to model these interactions and to account for them in the drive's controller. Unfortunately, accurate direct torque measurements are hardly feasible in operational conditions, as they often require a tailored mechanical design, or they affect the dynamics of the drivetrain. Several indirect measurement methods are therefore common in industrial practice, see e.g. $[1,2]$. A different approach, originating from research in noise control and receiving growing interest from other application fields, is virtual sensing [3]. This method estimates the desired torque information based on information from sensors that are less invasive, together with a dynamic model of the drivetrain. Extensive results have been shown in $[4,5]$ and references therein. These first developments resulted in a virtual torque sensing approach able to estimate the emulated torque up to $200 \mathrm{~Hz}$ with a dynamic range of $30 \mathrm{~dB}$ using a random walk model to describe the unknown input. A random walk model implies that little information about the estimated input is known. Yet, in many drivetrain applications we are aware of the presence of a certain periodicity of the load conditions. It is not uncommon for mechanical drivetrains to have torsional loading conditions that are periodic with respect to the rotation angle, e.g. the excitation of a crankshaft in a combustion engine. 
In order to validate novel research results on virtual torque sensing, including accounting for such periodicities, a lab-scale mechatronic powertrain emulator has been developed at the LMSD division of the KU Leuven Department of Mechanical Engineering [6]. It essentially consists of two electric motors that are coupled through a mechanical transmission. One of them is the actual drive, whereas the other one emulates the load dynamics of a real drivetrain. Obviously, the quality of this load emulation strongly depends on the performance of the load profile tracking control algorithm of the respective motor. The industrial standard for torque tracking in electric motors is proportional-integral (PI) control [7]. The real-time calculations these controllers require are limited, and they can thus run at high sampling rates. Moreover, the limited number of parameters (gains) makes them relatively easy to tune; even some physics-based analytical tuning guidelines have been derived $[8,9]$. If the motor interacts with a dynamical system with flexible modes (as is the case for the considered set-up), however, their performance generally becomes poor since these modes are not explicitly accounted for in the design. This work therefore presents the design of a linear feedback controller for this purpose through $\mathscr{H}_{\infty}$ loop shaping, based on an identified linear time-invariant (LTI) model. This model includes a flexible mode and still yields a model-based controller of a limited complexity that allows high sampling rates. Also the design of an inverse-based feedforward controller is considered.

The structure of this paper is as follows. First, the relevant components of the set-up of interest are described in more detail. The controller design approach including identification, closed-loop shaping and feedforward design is discussed next, followed by experimental results. The last section finally comprises concluding remarks and outlines future work.

\section{SET-UP DESCRIPTION}

Figure 1 depicts the set-up at hand. It has two three-phase induction motors with a rated power of $5.5 \mathrm{~kW}$ and rotational speeds up to 3000 revolutions per minute (RPM) within reach. Both of them are driven by a high-end digital rapid control prototype platform, updating high-level control loops at a rate of $16 \mathrm{kHz}$. The driving motor is velocity-controlled: its velocity control algorithm is assumed to be a given, as it has to reflect the drive of the powertrain to be emulated. Roughly speaking, closed-loop bandwidths of this control loop typically vary between 5 and $50 \mathrm{~Hz}$ for the applications that have been considered up to now. The second motor emulates the drivetrain dynamics and is supposed to accurately track a given (varying) torque profile. As a feedback signal for its torque control loop, a torque sensor with a torque limit of $50 \mathrm{Nm}$ is mounted between the torque-controlled motor and a double Cardan shaft that connects both motors. When assembled eccentrically, this shaft allows the generation of nonlinear dynamics if this would be

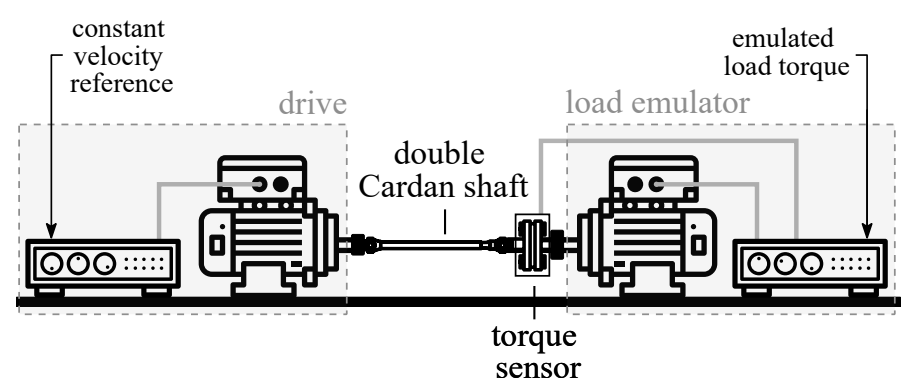

(A) CONCEPTUAL SCHEME.

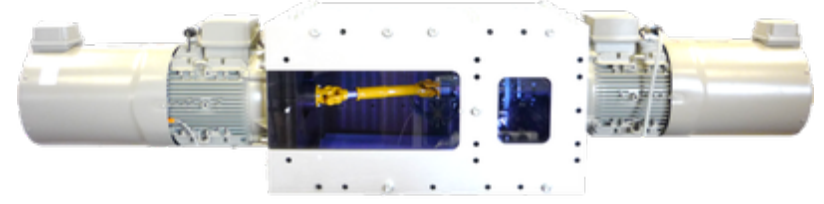

(B) LAB SET-UP.

\section{FIGURE 1. ILLUSTRATION OF THE DEVELOPED POWERTRAIN EMULATOR.}

useful for validation experiments. In the results that are presented in this work, however, both motors were always aligned and the universal joints were, thus, not exploited.

For more details on the set-up or a discussion of its other components (e.g. the frame), the interested reader is referred to [6].

\section{TORQUE CONTROLLER DESIGN Approach}

Figure 2 represents the considered control configuration. A standard error feedback configuration is opted for, comprising a linear single-input single-output (SISO) controller $K$ converting the torque tracking error $T_{\mathrm{e}}=T_{\mathrm{r}}-T$ into an appropriate quadrature current $i_{Q}$. Together with the direct current $i_{D}, i_{Q}$

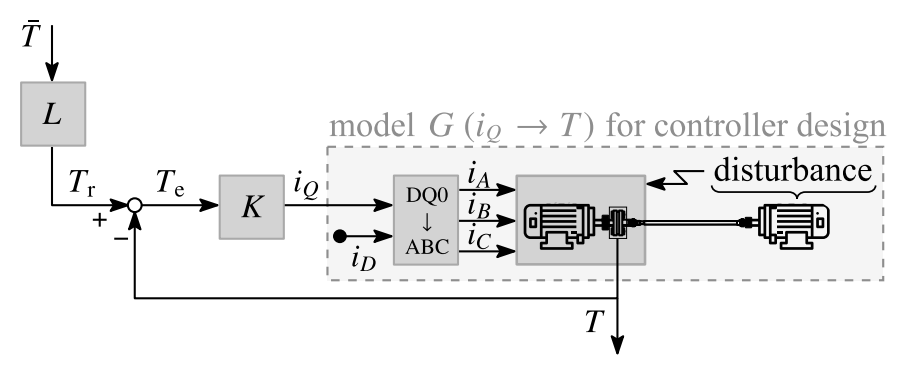

FIGURE 2. CONTROL CONFIGURATION SCHEME OF THE LOAD MOTOR AND THE VELOCITY-CONTROLLED DRIVE MOTOR. 
forms the basis for the underlying vector motor control, transforming them into the phase currents $i_{A}, i_{B}, i_{C}$. As is common practice, the direct current (and hence the magnetic flux) has been set to a constant value, so that $i_{Q}$ is the only remaining control signal. The low-level current control architecture has a limited relevance in the remainder of this paper, and will therefore not be discussed in more detail. With the feedback controller in place, the remaining closed-loop dynamics are compensated for by a torque reference (pre)filter $L$.

As explained earlier, the dynamics of the velocity-controlled driving motor and the torque-controlled load motor strongly interact with each other. That is, in the frequency range where both controllers are active, they will counteract and neither the constant velocity nor the torque reference will be properly tracked, unless the model of the given velocity controller is explicitly accounted for while designing the torque controller. For now, we do not consider such a 'coupled' design and instead interprete the effect of the velocity controller as an unknown disturbance (see Fig. 2). Hence, we expect the velocity controller to affect the response of the system $i_{Q} \rightarrow T$, further referred to as $G$, in the frequency range where its tracking performance is decent.

The synthesis of the control solution for the torque reference tracking is now divided into three steps. First, identification experiments are performed to obtain an LTI model of $G$. Then, a feedback controller is synthesized, based on the identified model, using closed-loop shaping in an $\mathscr{H}_{\infty}$ framework. Finally, an inverse-based feedforward controller is derived to improve the overall tracking performance.

\section{Identification}

Identification experiments are set up in a way that resemble operational conditions as close as possible, i.e. including disturbances from the velocity-controlled drive motor. Therefore, a constant velocity reference setpoint of 600 RPM is fed to the velocity controller of the drive motor, while a constant direct current reference of $i_{D}$ of $3 \mathrm{~A}$ as well as a constant quadrature current reference $i_{Q}$ of $3 \mathrm{~A}$ is sent to the load motor. On top of this constant $i_{Q}$, we add an excitation signal to identify $G$. The constant offset avoids torque reversals, that are known to introduce nonlinear effects and that are potentially harmful for the torque sensor for large amplitudes and/or high frequencies. The complexity level of the excitation signal itself is also limited due to hardware and memory constraints of the rapid control prototyping device. Therefore, a classic sine sweep with a linearly increasing frequency is selected. All modes from 0 up to $200 \mathrm{~Hz}$ are excited within a time frame of $200 \mathrm{~s}$, with an amplitude of $0.5 \mathrm{~A}$.

The same signals are applied several times for different tunings of the velocity controller of the drive motor to
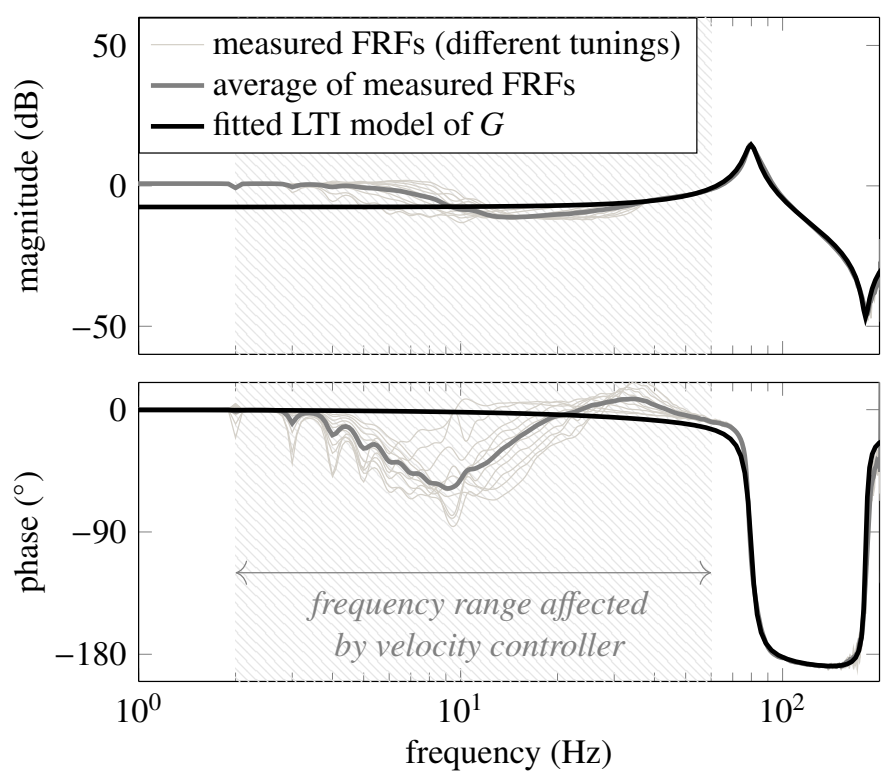

FIGURE 3. MEASURED AND IDENTIFIED FREQUENCY RESPONSE FUNCTIONS FROM $G$ FOR DIFFERENT VELOCITY CONTROLLER TUNINGS OF THE DRIVE.

investigate its effects on the load motor dynamics. Ten such tunings with varying tracking bandwidths up to approximately $50 \mathrm{~Hz}$ are considered. For every experiment, an $H_{1}$ transfer function estimate is calculated, as graphically shown by the thin grey ( $\square$ ) lines in the Bode diagram of Fig. 3. As expected, we clearly see the influence of the velocity controllers in the current-torque frequency response of the load motor. The region where this effect is considerable -especially in terms of the phase- is indicated by the hatched area, and is (not surprisingly) bounded by approximately $50 \mathrm{~Hz}$. At higher frequencies, a resonance/anti-resonance (R/AR) pair clearly appears, presumably originating from the rather compliant shaft couplings.

To reduce the influence of noise in the frequency range of interest, the different frequency response functions (FRFs) are averaged. An continuous-time LTI model structure with 3 poles and 2 zeros is proposed to fit the averaged FRF accurately; the R/AR pair in particular. A weighted nonlinear least-squares error minimization method [10] yields us the unknown transfer function coefficients. The weighting factors are chosen to be equal for all excited frequencies, except for the range that is thoroughly affected by the velocity controller of the drive motor (i.e., the hatched region in Fig. 3). Accepting a potential loss of torque tracking performance in this band caused by model inaccuracies, the weighting factors are set to 0 here. The frequency response of the resulting model is drawn as a black (匹) line in Fig. 3. 


\section{Feedback controller design}

For obtaining an appropriate linear feedback controller, we translate the desired specifications to weighting filters that are used to enforce particular shapes on relevant closed-loop input-output transfers (closed-loop shaping). By solving a mixed-sensitivity/multi-objective problem, every separate specification can be rather intuitively mapped to an $\mathscr{H}_{\infty}$ constraint, especially for SISO cases like the problem at hand.

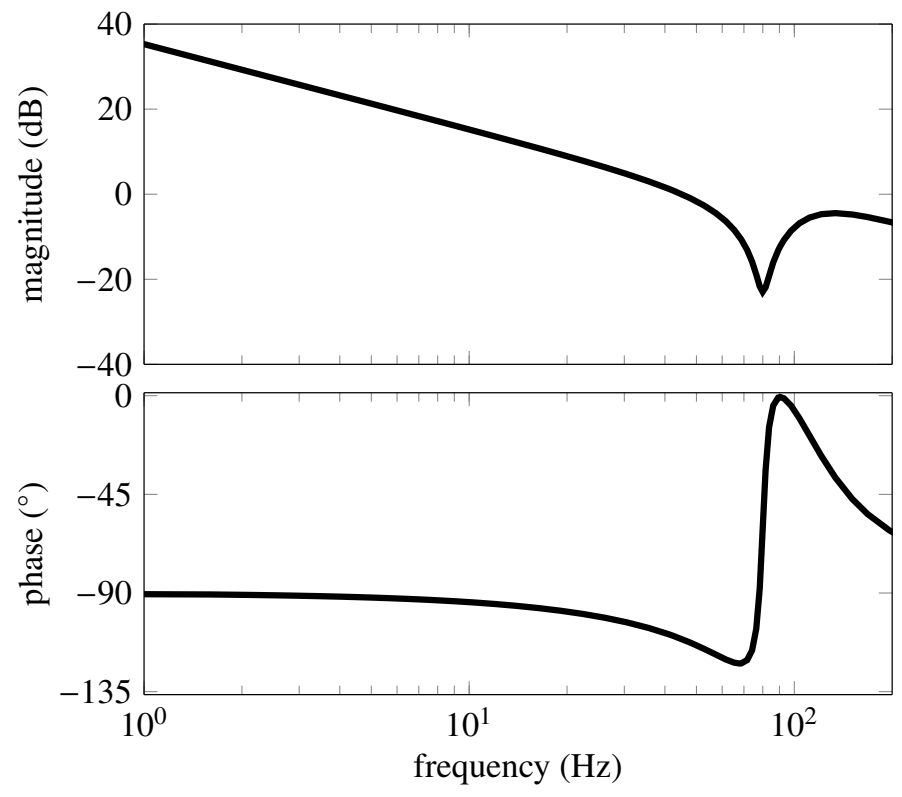

FIGURE 4. FREQUENCY RESPONSE OF THE CONTROLLER THAT SOLVES PROBLEM (1).
From here, we follow a standard approach that, for example, has been explained in great detail in [11] and references therein. In short, we select the so-called sensitivity $S=T_{\mathrm{e}} / T_{\mathrm{r}}$ and the input sensitivity $U=i_{Q} / T_{\mathrm{r}}$ as the closed-loop transfers to be shaped. Effective tracking and proper disturbance rejection, as a first objective, then corresponds to low-frequency roll-off of $S$, and is imposed by selecting a weighting filter $W_{S}(s)=\omega_{\mathrm{bw}} / s ; \omega_{\mathrm{bw}}$ representing the targeted control bandwidth. If we constrain $\left\|W_{S} S\right\|_{\infty} \leq \gamma(1 \mathrm{a})$, then minimizing $\gamma$ corresponds to maximizing this bandwidth. Similarly, and as a second design objective, a desired minimal amount of damping of the closed-loop response is acquired by constraining the peak gain of $S$ to a design value $1 / M_{S}$, so that $\left\|M_{S} S\right\|_{\infty} \leq 1$ (1b). As a third and last specification, we enforce high-frequency roll-off on $U$ to avoid large control signals, especially at high frequencies, and to suppress noise. An appropriate weighting filter to include in the constraint $\left\|W_{U} U\right\|_{\infty} \leq 1$ (1c) for this purpose is $W_{U}(s)=s / \omega_{\mathrm{bw}}=W_{S}^{-1}(s)$. In summary, we solve for the feedback controller $K$ that solves the following optimization problem (1):

$$
\begin{array}{ll}
\underset{K, \gamma}{\operatorname{minimize}} & \gamma \\
\text { subject to } & \left\|W_{S} S\right\|_{\infty} \leq \gamma \\
& \left\|M_{S} S\right\|_{\infty} \leq 1 \\
& \left\|M_{U} U\right\|_{\infty} \leq 1 \\
& \text { closed-loop stability }
\end{array}
$$

The numeric values of the design parameters were chosen as $\omega_{\mathrm{bw}}=100 \cdot 2 \pi \mathrm{rad} / \mathrm{s}$ and $M_{S}=0.5=1 /(6 \mathrm{~dB})$.
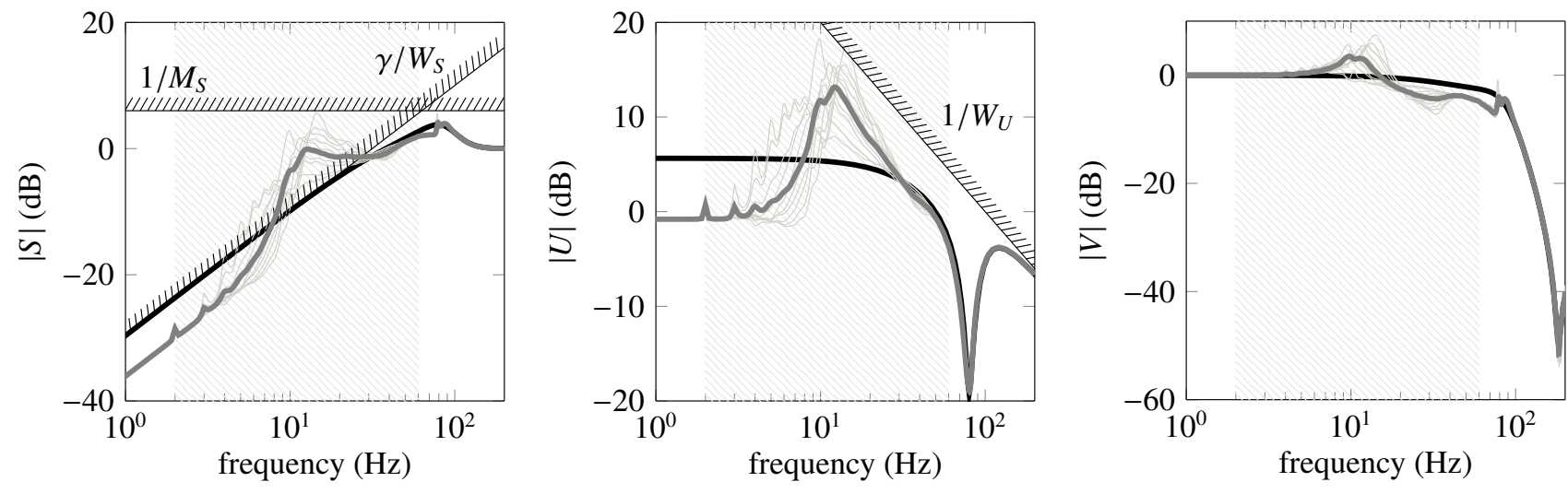

FIGURE 5. SHAPED CLOSED-LOOP TRANSFERS $S=T_{\mathrm{e}} / T_{\mathrm{r}}, U=i_{Q} / T_{\mathrm{r}}$ AND $V=T / T_{\mathrm{r}}$ RESULTING FROM THE SOLUTION OF PROBLEM (1), TOGETHER WITH VISUALIZED CONSTRAINTS. THE DIFFERENT LINES CORRESPOND TO THE MODELS SHOWN IN FIG. 3. 
Several solution methods for problem (1), which is a high-level formulation of a generally complicated optimization problem, have been developed in the control systems community over the past decades. Well-known methods involve reformulations in terms of BMIs, LMIs, Riccati equations, polynomial factorizations, etc. Manual transcription of the high-level formulations into these mathematical representations is a tedious and time-consuming job. To shield the practising control engineering from this issue, a supporting open-source toolbox for MATLAB has been developed: LCToolbox [12]. Problem (1) is directly parsed in this toolbox, returning the controller that is depicted in Fig. 4 and that realizes the loop shapes of Fig. 5. For the sake of completeness, this figure also shows the complementary sensitivity $V=T / T_{\mathrm{r}}$, as it is an appropriate transfer to assess the closed-loop performance, along with $S$ and $U$. The closed-loop shapes of the latter two clearly follow the desired loop shapes, i.e. the shapes of the inverted weighting filters. We see that the corresponding controller contains an integrator and compensates for the resonance of the system. On the contrary, the anti-resonance is not compensated for. This would require large control signals, while the input sensitivity was conflictingly forced to steeply roll off.

The continuous-time controller is discretized using a standard bilinear transformation (Tustin's method) for implementation on the control platform of the load motor. Due to the high sampling rate of $16 \mathrm{kHz}$, this discretization hardly affects the frequency response of the controller and its behavior.

\section{Feedforward controller design}

In order to further improve the tracking performance, the torque reference signal $T_{\mathrm{r}}$ of the feedback loop can be shaped to account for the remaining dynamics of the closed-loop transfer $V$. That is, $T_{r}$ is the result of prefiltering the actual desired torque reference $\bar{T}$ with a feedforward prefilter $L$, see Fig. 2. A parametric version and a non-parametric implementation of this filter $L$ are possible, and are both considered hereafter.

Shaping $T_{\mathrm{r}}$ from $\bar{T}$ based on parametric model inversion is straightforward. The LTI model of $V=G K(1+G K)^{-1}$ is first inverted and then extended with a low-pass filter $Q$ to avoid contributions of the uncertain high-frequency characteristics of the model. As the parametric model of $G$ is only reliable up to $200 \mathrm{~Hz}$ (the highest frequency that was considered in the identification), the cut-off frequency of this (standard, e.g. Butterworth) filter $Q$ is chosen as such. It is noteworthy that $Q$ can also be chosen acausal (and therefore not necessarily introducing phase lag) if the trajectory of $\bar{T}$ is known in advance. In summary, we have $L=Q(1+G K)(G K)^{-1}$.

Non-parametric model inversion is similar to parametric model inversion, but omits the model fitting step. Instead, an $H_{1}$ transfer function estimate of $V$ is identified (in closed-loop) in a similar fashion as the model of $G$ (cf. supra). The reliable part of its frequency response is then directly inverted and multiplied with the spectrum of $\bar{T}$. The uncertain part of the inverse frequency response is set to 0 ; the non-parametric analog of the low-pass filter $Q$ in the parametric version. The inverse discrete Fourier transform (DFT) of the resulting spectrum immediately yields the shaped reference $T_{\mathrm{r}}$ without having introduced model fitting errors. Note that this technique, as opposed to its parametric variant with a causal filter $Q$, can only be applied if $\bar{T}$ is completely known upfront. Thus, sensu stricto, $L$ is no longer a filter in this case.

\section{EXPERIMENTAL RESULTS Experiment description}

Drivetrain load dynamics are often periodic if the drive motor realizes a constant rotational velocity. This situation emulates, for instance, the classic case of a reciprocating cam system. As an experimental validation of the proposed control scheme, we generate several such periodic torque load profiles to be tracked by the load motor while the drive motor runs at various constant rotational speeds in the range of 300 RPM to 1900 RPM. All of these experiments show a highly comparable tracking performance. For the sake of compactness, the authors therefore only report on the results of one sample experiment, which is graphically summarized in Fig. 6 and described in more detail in the next section.

Other experiments have indicated a decreasing torque tracking performance for typical references at rotational speeds lower than 300 RPM due to disturbing control actions of the velocity controller, and at rotational speeds higher than 1900 RPM due to the limited control bandwidth. Furthermore, the torque amplitude shall not substantially deviate from the range of amplitudes of the excitation signals during the identification. Indeed, leaving the operational range that was modeled by linear dynamics was found to significantly deteriorate the performance.

\section{Discussion}

The solid black line (घ) of Fig. 6(A) shows one period of the reference torque profile $\bar{T}$ of the presented experiment, performed at a rotational velocity of 600 RPM. It varies between 3 and $6 \mathrm{Nm}$ and has a duration of one shaft revolution. The torque evolution is therefore expressed in terms of the drive motor angle (lower labels), but can be equivalently expressed in terms of time (upper labels). The calculated parametric and nonparametric feedforward signals $T_{\mathrm{r}}$ corresponding to $\bar{T}$ are also plotted in this figure, in dark grey ( $\square$ ) and light grey ( $\square$ ) respectively. $T_{\mathrm{r}}$ clearly leads in time with respect to $\bar{T}$, and also noticeably contain contributions at approximately $180 \mathrm{~Hz}$, the anti-resonance frequency of $V$ (see Fig. 5). 
Figure 6(B) depicts the actual torque measurements $T$ of 30 revolutions ( $3 \mathrm{~s}$ ), on top of each other, realized by the feedback control loop for the references $T_{\mathrm{r}}$ of (A). The same color convention applies, and $\bar{T}$ is plotted once more to assess the tracking performance. It appears that $T$ is not only very repeatable throughout the different cycles, but also properly tracks $\bar{T}$ for both references $T_{\mathrm{r}}$. However, the nonparametric method slightly outperforms its parametric counterpart, as can be seen at about $90^{\circ}(25 \mathrm{~ms})$. This observation becomes more visible on Fig. 6(C), showing the difference of both curves with the reference $\bar{T}$, or by calculating average values of the tracking errors over time, as indicated by Tab. 1 . The root-mean-square (RMS) error (2-norm) as well as the 1-norm and $\infty$-norm are larger for the parametric feedforward than for the nonparametric one.

(A)

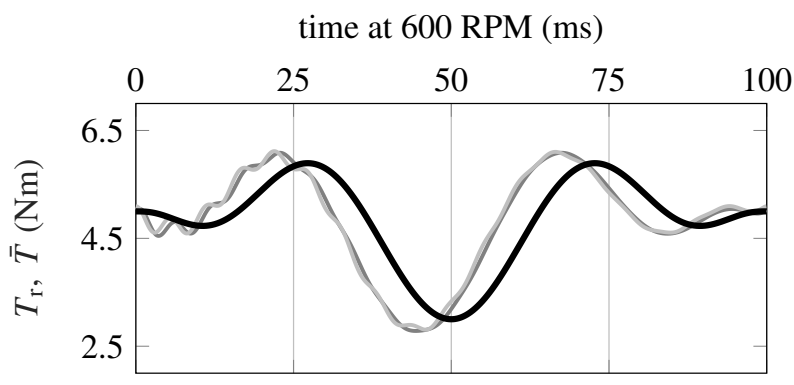

(B)

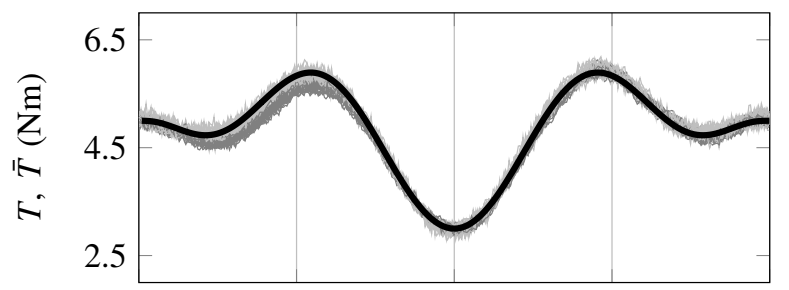

(C)

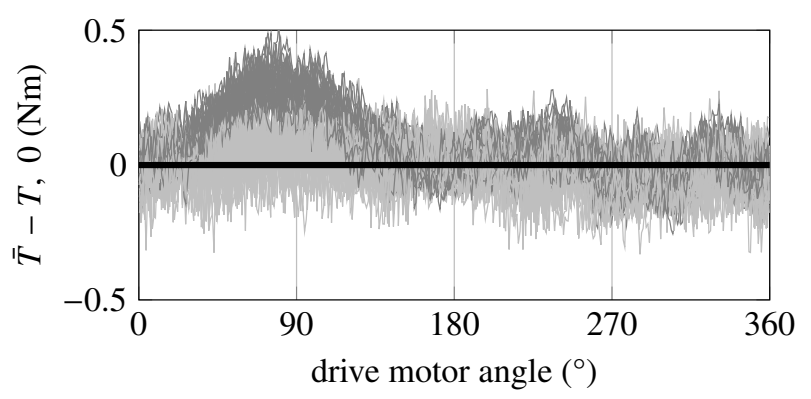

- feedback + parametric feedforward
- feedback + nonparametric feedforward
- desired torque profile $\bar{T}$

FIGURE 6. EXPERIMENTAL RESULTS OF TRACKING 30 PERIODS OF A PERIODIC TORQUE REFERENCE AT A CONSTANT ROTATIONAL REFERENCE SPEED OF 600 RPM.
To explain the slight differences between the usage of the nonparametric and the parametric feedforward, we refer back to the identification step of the parametric model $G$ earlier. Indeed, the lower frequency range was -deliberately- not adequately fitted because of the dependence of the velocity controller characteristics on the envisioned drivetrain emulation. On the other hand, the nonparametric model of $V$ was identified with the velocity controller that was also active during the experiments. Thus, the nonparametric model is definitely more accurate, particularly for references $\bar{T}$ that contain relatively low frequencies.

\section{CONCLUSION AND FUTURE WORK}

This paper reported on the details of a design procedure of a linear control system for a torque tracking problem of a lab-scale powertrain emulator. An LTI model including a flexible mode was identified and formed the basis of a frequency domain design procedure of a feedback controller by means of $\mathscr{H}_{\infty}$ optimization. A prefilter was proposed to compensate for the remaining closed-loop dynamics in a parametric and a nonparametric way. Experimental results demonstrated the effectiveness of the approach.

Two different potential improvements are planned to be studied in the future. First, it has become clear from the experiments that explicitly accounting for the dynamics of the velocity controller of the drive motor may yield a significant performance gain. However, this would require time-consuming steps like model identification and feedback controller tuning for every emulation. A possible solution consists of parametrizing the dependency on the velocity controller in terms of its characteristic parameters. In industrial practice, for example, the velocity controllers are often PID. Then, by identifying the model $G$ as a function of these three parameters as proposed in [13], one can design a linear-parameter varying (LPV) torque feedback controller depending on these same parameters [14]. Second, since the torque profiles are typically periodic, repetitive control (RC) can improve the tracking performance of the feedback controller by explicitly relying on

TABLE 1. AVERAGE TRACKING ERRORS OVER TIME IN THE SAMPLE EXPERIMENT.

\begin{tabular}{lccc}
\hline $\bar{T}-T(\mathrm{mNm})$ & $\|\cdot\|_{1}$ & $\|\cdot\|_{2}$ & $\|\cdot\|_{\infty}$ \\
\hline $\begin{array}{l}\text { nonparametric model inversion } \\
\text { for calculation of } T_{\mathrm{r}}\end{array}$ & 90 & 71 & 337 \\
$\begin{array}{l}\text { parametric model inversion for } \\
\text { calculation of } T_{\mathrm{r}}\end{array}$ & 140 & 103 & 525 \\
\hline
\end{tabular}


the periodicity of the input [15]. As a final remark, we note that iterative learning control (ILC) is likely to shape a better reference signal for the feedback control loop in comparison with a prefilter. Nevertheless, it requires several time-consuming experiments for every specific reference, and is therefore not considered as practically feasible for this application.

\section{ACKNOWLEDGMENT}

This research is partially supported by Flanders Make projects: SBO ROCSIS: Robust and Optimal Control of Systems of Interacting Subsystems, SBO MoForM: Model-based Force Measurements, ICON DTDesign: Framework for Systematic Design of Digital Twins, and INFRA I3oT: Infrastructure for Industrial Internet of Things. This work also benefits from KU Leuven project C14/15/067: B-spline based certificates of positivity with applications in engineering.

\section{REFERENCES}

[1] Beihoff, B., 1996. "A survey of torque transduction methodologies for industrial applications". In 1996 Annual Pulp and Paper Industry Technical Conference (PPIC), Birmingham, USA.

[2] Garshelis, I., 1999. The Measurement, Instrumentation, and Sensors: Handbook. Electrical Engineering Handbook Series. CRC Press, ch. 24: 'Torque and Power Measurement'.

[3] Lichuan Liu, Kuo, S. M., and Zhou, M., 2009. "Virtual sensing techniques and their applications". In 2009 International Conference on Networking, Sensing and Control (ICNSC), Okayama, Japan.

[4] Forrier, B., Naets, F., and Desmet, W., 2018. "Virtual torque sensing: A model-based approach for indirect measurement of dynamic operational loads on mechatronic powertrains". PhD thesis, KU Leuven.

[5] Forrier, B., Naets, F., and Desmet, W., 2018. "Broadband load torque estimation in mechatronic powertrains using nonlinear kalman filtering". IEEE Transactions on Industrial Electronics, 65(3), pp. 2378-2387.

[6] Forrier, B., Boonen, R., and Desmet, W., 2016. "Development of a novel test setup for validation of virtual sensing on mechatronic drivetrains". In International Conference on Powertrain Modelling and Control (PMC), Loughborough, UK.

[7] Olarinoye, G. A., Akinropo, C., Atuman, G. J., and Abdullahi, Z. M., 2019. "Speed control of a three phase induction motor using a PI controller". In 2019 2nd International Conference of the IEEE Nigeria Computer Chapter, pp. 1-7.

[8] Terörde, G., 2004. Electrical Drives and Control Techniques. ACCO Uitgeverij.
[9] Alfeu, J., Filho, S., and Ruppert, E., 2010. "Tuning PI regulators for IM direct torque control using complex transfer function". In 2010 9th IEEE/IAS International Conference on Industry Applications - INDUSCON 2010, pp. 1-6.

[10] Pintelon, R., and Schoukens, J., 2012. System Identification: A Frequency Domain Approach, 2nd Edition. Wiley-IEEE Press.

[11] Skogestad, S., and Postlethwaite, I., 2005. Multivariable Feedback Control: Analysis and Design, 2nd Edition. John Wiley \& Sons, Inc.

[12] Swevers, J., Jacobs, L., Singh, T., Turk, D., Verbandt, M., and Pipeleers, G., 2020. "LCToolbox: Facilitating optimal linear feedback controller design”. IEEJ Journal of Industry Applications, 9(2), pp. 109-116.

[13] Turk, D., Jacobs, L., Singh, T., Decré, W., and Swevers, J., 2019. "Identification of linear parameter-varying systems using B-splines". In 2019 European Control Conference (ECC), Naples, Italy.

[14] Hilhorst, G., Lambrechts, E., and Pipeleers, G., 2016. "Control of linear parameter-varying systems using Bsplines". In $55^{\text {th }}$ IEEE Conference on Decision and Control (CDC), Las Vegas, USA.

[15] Pipeleers, G., Demeulenaere, B., and Swevers, J., 2010. Optimal Linear Controller Design for Periodic Inputs. Lecture Notes in Control and Information Sciences. Springer. 